This item was submitted to Loughborough's Research Repository by the author.

Items in Figshare are protected by copyright, with all rights reserved, unless otherwise indicated.

\title{
Handgrip strength, inflammatory markers and mortality
}

PLEASE CITE THE PUBLISHED VERSION

https://doi.org/10.1111/sms.13433

PUBLISHER

(c) Wiley

\section{VERSION}

AM (Accepted Manuscript)

\section{PUBLISHER STATEMENT}

This is the peer reviewed version of the following article: SMITH, L., YANG, L. and HAMER, M., 2019. Handgrip strength, inflammatory markers and mortality. Scandinavian Journal of Medicine \& Science in Sports, 29 (8), pp.1190-1196, which has been published in final form at https://doi.org/10.1111/sms.13433. This article may be used for non-commercial purposes in accordance with Wiley Terms and Conditions for Use of SelfArchived Versions.

\section{LICENCE}

CC BY-NC-ND 4.0

\section{REPOSITORY RECORD}

Smith, Lee, Lin Yang, and Mark Hamer. 2019. "Handgrip Strength, Inflammatory Markers and Mortality". figshare. https://hdl.handle.net/2134/37593. 
$4{ }^{1}$ The Cambridge Centre for Sport and Exercise Sciences, Anglia Ruskin University,

5 Cambridge; ${ }^{2}$ Department of Epidemiology, Medical University of Vienna, Austria; ${ }^{3}$ School

6 Sport, Exercise Health Sciences, Loughborough University, Loughborough, UK

7

8 *Correspondence: Dr Lee Smith; ${ }^{1}$ The Cambridge Centre for Sport and Exercise Sciences,

$9 \quad$ Anglia Ruskin University, Cambridge, UK; lee.smith@anglia.ac.uk

10 Word count $=1,899$

11 Running Head: handgrip strength and mortality

12 Disclosures: The authors declare no conflicts of interest. 
Abstract

Purpose: To investigate the extent to which inflammatory markers explain the association between handgrip strength and mortality.

Methods: Analyses of data from The English Longitudinal Study of Ageing. Handgrip strength and inflammatory marker data (C-reactive protein and Fibrinogen) were collected at baseline (2004/5) and inflammatory marker data at follow up (2012/13). Participant data were linked with death records. General linear models were used to explore associations between handgrip strength and inflammatory markers at follow up. Cox proportional hazards regression models were used to examine associations between grip strength and risk of death. Models were estimated with the covariates age, sex, wealth, physical activity, smoking, depressive symptoms, long standing illness, adiposity.

Results: The sample comprised 5,240 participants (mean age 65.9 (SD 9.4) yrs; 53.8\% female). Over an average $9.7 \pm 1.4$ yrs follow up there were 650 deaths. Inverse associations were evident between handgrip strength and change in inflammatory markers in women only. There was an association between grip strength and lower risk of mortality in women (hazard ratio $=0.85 ; 95 \% \mathrm{CI}, 0.74,0.98)$ after adjusting for age and wealth. The association was attenuated after adjustment for clinical and behavioural risk factors $(0.92 ; 0.79,1.07)$, and further attenuated after adjusting for inflammatory markers $(0.95 ; 0.82,1.11)$.

Conclusion: Higher grip strength is associated with lower levels of inflammation at 8-year follow-up, and inflammatory markers partly explained the association between handgrip strength and mortality.

Keywords: Handgrip strength, mortality, inflammatory markers, ELSA 
Physical activity promotes cardiorespiratory fitness and the maintenance of lean muscle mass with ageing. ${ }^{1}$ The health benefits of cardiorespiratory fitness are well established ${ }^{2}$ although less is known about muscle strength..$^{3,4}$

Handgrip strength is a valid measure of physical function/ performance that has been widely used in observational cohort studies and clinical settings. ${ }^{5-8}$ Several studies have shown that handgrip strength is inversely associated with risk of mortality ${ }^{9-11}$ although the mechanisms linking muscle strength and disease outcomes is poorly understood. One possible explanation is systemic inflammation. Systemic inflammation has been shown to be associated with a plethora of noncommunicable diseases (e.g. see, Ridker et $\mathrm{al}^{12}$ and Sin et $\mathrm{al}^{13}$ ).

Three well studied inflammatory biomarkers include Interleukin 6 (IL-6), C-reactive protein (CRP), and Fibrinogen. Briefly, IL-6 is a pleiotropic cytokine that is secreted by T cells and macrophages to activate immune responses during infection or after trauma (tissue injury). ${ }^{14}$ CRP is produced in the liver and it is an acute phase reactant thus levels will rise when inflammation is present. ${ }^{15}$ Finally, Fibrinogen is a blood plasma protein that is made in the liver. It is also an acute phase reactant and levels rise when inflammation is present. ${ }^{16}$ Unfavourable levels of these three inflammatory biomarkers have been found to be associated with several physical and mental health conditions. ${ }^{17-19}$

Inflammatory processes have also been linked with muscle atrophy (and thus weaker grip strength), ${ }^{20}$ and muscle tissue expresses various transcriptional co-activators that may promote systemic anti-inflammatory effects. ${ }^{21}$ We have previously shown cross-sectional associations between systematic inflammation and muscle strength, ${ }^{22}$ consistent with others. ${ }^{23}$ In another study, higher levels of IL-6 and CRP were associated with a two- to three-fold greater risk of losing more than $40 \%$ of grip strength over 3 years follow-up. ${ }^{24}$ The current 
67 literature therefore suggests that inflammation is associated with muscle strength although the direction of the association is not clear.

69

We hypothesized that inflammatory processes might in part explain the association between handgrip strength and mortality. The primary aim of the present study was to investigate the longitudinal association between handgrip strength and inflammatory markers, and examine the extent to which inflammatory markers explain the association between handgrip strength and mortality.

\section{METHODS}

\section{Study sample and procedures}

The English Longitudinal Study of Ageing (ELSA) is a cohort study of older adults previously described. ${ }^{25}$ The sample was drawn from participants in the Health Survey for England (HSE), an annual cross-sectional survey that is designed to monitor the health of the general population. For wave 1, participants were recruited from the HSE in 1998, 1999 and 2001. Eligibility criteria included, membership of a participating household from HSE in which at least one person had agreed to follow-up, born before 1 March 1952 and living in a private household in England at the time of the first wave of fieldwork. Data on grip strength and inflammatory markers was first collected at wave $2(2004 / 5)$, and was thus used as the baseline for the present analyses. Inflammatory marker data collection were repeated at wave $6(2012 / 13)$ in survivors. Participants gave full informed consent to participate in the study. Ethical approval was obtained from the London Multi-center Research Ethics Committee, compliant with the Declaration of Helsinki.

\section{Clinical assessments}


90 Anthropometric data (weight, waist, hip), grip strength and blood samples were collected by trained nurses. Participants were excluded from providing blood if they reported clotting and bleeding disorders, or taking anti-coagulant medication, and excluded from hand grip tests if they had swelling or inflammation, severe pain, or a recent injury or surgery to the hand in the preceding 6 months. Handgrip strength $(\mathrm{kg})$ of the dominant hand was assessed using the Smedley hand-held dynamometer (Stoelting Co, IL, USA ), using the average of three measurements. Participants were required to hold the device at a right angle to their body and exert maximum force for a couple of seconds when instructed. Successive trials were alternated between dominant and non-dominant hands. Body weight was measured without shoes and in light clothing using Tanita electronic scales. Waist circumference was recorded twice midway between the iliac crest and lower rib and hip circumference around the widest portion of the buttocks using measuring tape. Central obesity was defined using waist to hip ratio (WHR) World Health Organization criteria (WHR $\geq 0.85$ in women and WHR $\geq 0.90$ in men). Blood samples were analyzed for high sensitivity C-reactive protein (CRP) and fibrinogen, described elsewhere. ${ }^{26}$

\section{Mortality}

The individual participant data were linked with death records from National Health Service registries for all consenting respondents (96.5\% of the sample) up to February 2012.

\section{Covariates}

Trained interviewers asked questions on cigarette smoking (current, ex-smoker or nonsmoker), frequency of vigorous, moderate- and low-intensity physical activity ( $>$ once a week, once a week, $1-3$ times a month, and hardly ever/never), chronic illnesses (at least one vs. none), and depressive symptoms (using a score $>3$ on the 8-item Centre of 
115 Epidemiological Studies Depression scale). ${ }^{27}$ Wealth was self-reported, comprising of the total value of the participant's home (excluding mortgage), financial assets such as savings, business assets, and physical wealth such as artwork or jewelry, which has been shown to best capture the material resources available to older adults. ${ }^{28}$ Wealth was grouped into quintiles relative to the ELSA sample.

\section{Statistical analysis}

Handgrip was standardised for body mass (as the two variables were moderately correlated, $\mathrm{r}>0.40$ ) and treated as a continuous variable using the standardised score. Inflammatory markers were examined for normality and log transformed where appropriate. In all analysis we ran models separately for men and women as handgrip strength was greater in men. We used general linear models to explore longitudinal associations between handgrip strength and change in inflammatory markers. Models were adjusted for age, sex, levels of inflammatory marker at baseline, wealth, physical activity, smoking, depressive symptoms, long standing illness, WHR.

Cox proportional hazards regression models were employed to examine associations between grip strength and death. Years were the time scale for the follow-up calculated from age at death, and for participants with no record of an event, the data were censored at February 2012. Plots of the Nelson-Aalen cumulative hazard estimates were examined to assess the proportional hazards assumption. We estimated models that initially contained the covariates age, sex, wealth (model 1), with further adjustment for physical activity, smoking, depressive symptoms, long standing illness, WHR (model 2). These covariates were considered as confounders and selected a priori based on previous literature. ${ }^{29-31} \mathrm{We}$ then added inflammatory markers (log CRP, fibrinogen) as continuous variables to theoretically test for mediation. In these mediation analyses we primarily focused on the change in effect estimate 
and confidence intervals as "statistical significance" is less relevant. ${ }^{33}$ In sensitivity analyses we removed participants with activity limiting illnesses to examine if reverse causation was driving the results. All analyses were conducted using SPSS version 22 (SPSS, Chicago, IL).

\section{RESULTS}

144 At baseline 5,812 participants provided a blood sample and data on hand grip strength. After removing participants not consenting to mortality linkage $(n=138)$ and those with missing covariates $(n=434)$ the analytic sample for survival analyses comprised 5,240 participants (mean age 65.9 (SD 9.4) yrs; $53.8 \%$ female). In the longitudinal analyses that modelled change in inflammatory markers we further excluded participants that died through follow up $(n=650)$ and those that did not provide blood samples at 8 years follow up $(n=1,885)$, leaving an analytic sample of 2,705 participants (age $63.3 \pm 7.8 \mathrm{yrs} ; 55.2 \%$ female).

At baseline the mean handgrip strength of dominant hand was $38.4 \pm 9.7 \mathrm{~kg}$ (men) and $22.5 \pm 6.6 \mathrm{~kg}$ (women); normalised for body mass $0.47 \pm 0.12$ (men) and $0.33 \pm .10$ (women). Participants in the highest hand grip strength tertiles were younger, more vigorously active, more affluent, reported less illness and depressive symptoms, and recorded lower prevalence of central obesity (Table 1).

In longitudinal analyses that examined baseline grip strength and change in inflammatory markers over 8 years follow up inverse associations were evident with both fibrinogen and CRP in women only (Table 2). This was the case in fully adjusted analyses and also among the pooled sample of men and women. In men, the inverse associations between grip strength and change in inflammatory markers were evident in age adjusted models but did not persist in models adjusted for all covariates. 
Over an average $9.7 \pm 1.4 \mathrm{yrs}$ follow up there were 650 deaths. There was an association between baseline grip strength and lower risk of mortality in women (hazard ratio [per SD increase in grip strength] $=0.85 ; 95 \% \mathrm{CI}, 0.74,0.98$ ) after adjusting for age and wealth; no associations were seen in men $(0.94 ; 0.83,1.06)$ (Table 3$)$. The association was somewhat attenuated after adjustment for clinical and behavioural risk factors, and further attenuated after adjusting for inflammatory markers. In mutually adjusted models the covariates associated with mortality included longstanding illness, physical inactivity, smoking, and CRP (Table S1, SDC, association between clinical and behavioural covariates with mortality).

In sensitivity analyses we repeated the survival models after removal of participants who reported that their illness/disability limited their activity $(n=1,409)$. The effect estimates remained largely unchanged albeit confidence intervals became wider as a result of reduced numbers (Table S2, SDC, the extent to which baseline clinical and biological factors explain associations between handgrip and mortality (excluding participants with activity limiting illnesses at baseline)).

\section{DISCUSSION}

In this population-based sample of older English adults, those with higher levels of grip strength were significantly more likely to have lower levels of systemic inflammatory markers, CRP and Fibrinogen, at follow-up. This association was seen in females, only. Grip strength was inversely associated with risk of mortality in females only. However, this association was attenuated to the null after adjustment for clinical and behavioural risk factors, and further attenuated after adjusting for inflammatory markers.

Our findings support previous research on the association between grip strength and inflammatory markers ${ }^{34,35}$ and add to this body of work by showing that longitudinal 
associations persist when controlling for a range of clinical and behavioural risk factors including measures of central adiposity.

One plausible explanation for the association between grip strength and inflammation is that muscle mass is a predictor of greater grip strength and those who partake in regular exercise tend to have greater muscle mass. Exercise using large muscle groups produces a short-term, inflammatory response, and both cross-sectional comparisons and longitudinal exercise training studies demonstrate a long-term "anti-inflammatory" effect. ${ }^{36}$ Models in the present study controlled for levels of physical activity, though the types of activities (aerobics vs. resistance training) were not clearly separated. One other possible explanation is that high levels of circulating inflammatory markers and/or cytokines are associated with low muscle mass per se in older and obese people thus suggesting that muscle itself my play a role in regulating inflammatory markers. ${ }^{37-39}$

It is feasible that inflammatory markers could be exercising their effect through a combination of lifestyle and behavioural factors; indeed adding inflammatory markers directly to Model 1, (Table 3 ) in women closely mimicked (HR=0.91; 95\% CI, $0.79-1.05)$ the effect of adding lifestyle / behavioural covariates. The association of grip strength with inflammation and mortality was more robust in females, which is consistent with other findings. ${ }^{22,40}$ ). Recent meta-analytic data showed grip strength was associated with reduced mortality in both genders, although the association was stronger in women. ${ }^{41}$ Our sex specific findings are unremarkable as the aging process particularly affects women, such that women are more susceptible to sarcopenia than men. ${ }^{42,43}$ In our own sample, the proportion of men having grip strength above the gender specific sarcopenia diagnosis cut-off is higher $(90.5 \%>=26 \mathrm{~kg})$ than that of women $(84.8 \%>=16 \mathrm{~kg}) .{ }^{44}$ Furthermore, male and female hormones modulate the immune system differently, thus resulting in differential age-related 
pathologies. ${ }^{45}$ Therefore, muscle strength might be particularly important for women in regulating chronic inflammation. ${ }^{46}$

Clear strengths of the present study include the large population-based sample of older English adults, the longitudinal design, the objective measure of both the exposure and the outcome, and repeated measure of inflammatory biomarkers. However, the data must be interpreted considering the following limitations. Data on skeletal muscle mass were not available in the present cohort and we relied on measurements of muscle strength alone. While lean mass and strength (muscle quality) may not decline at the same rate, loss of lean mass is strongly associated with strength decline in both men and women. ${ }^{47}$ In our study, grip strength was standardized for body weight in the analyses. This approach allows us to account for muscle strength driven by body composition. There are ongoing discussions on how to standardize the procedure and utilization of grip strength measures in clinical and epidemiological studies, particularly in the ageing population. ${ }^{48}$ Although a unified method is yet to be developed, our approach of body weight standardized grip strength has shown to be a superior technique in the aging population. ${ }^{49,50}$ We were unable to account for underlying disease that were not detected through our measures; however, the results were largely unchanged after excluding participants with activity limiting illnesses suggesting reverse causation is an unlikely interpretation of our data. Participants retained in our analyses were generally healthier than the overall sample (driven by our exclusion criteria for blood and handgrip tests), thus our results are likely to reflect a conservative estimate of the true associations. Since the data are observational we cannot exclude the possibility of residual confounding. Nevertheless, recent evidence using Mendelian randomisation approaches suggested genetic correlations of grip strength with cardiometabolic traits and markers of frailty. ${ }^{51}$ Our study provides evidence on plausible biological pathways linking strength and mortality thus further strengthening causal inferences. 


\section{Perspective}

236 The present longitudinal analyses suggest that higher grip strength is associated with lower

237 levels of inflammation at 8-year follow-up, and inflammatory markers partly explain the association between handgrip and mortality. Interventions designed to prevent a decline in physical functioning in females, especially, may improve inflammatory profiles and health. Such interventions may want to focus on functional task exercises as opposed to resistance exercises as functional task exercises have been shown to be more effective at improving functional task performance. Moreover, other literature suggests that functional task exercises may have an important role in helping maintain an independent lifestyle. ${ }^{52}$

\section{Authors contribution Statement}

245 5

Hamer is the guarantor; he conceptualized and designed the study, performed statistical analyses, and approved the final manuscript as submitted. Smith conceptualized and designed the study, drafted the initial manuscript, and approved the final manuscript as submitted; Yang conceptualized and designed the study, provided critical revision of the manuscript and approved the final manuscript as submitted.

\section{Conflicts of Interest}

The authors have no conflicts of interest. The results of the present study do not constitute endorsement by ACSM and are presented clearly, honestly, and without fabrication, falsification, or inappropriate data manipulation

(1)

6

.

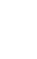




\section{References}

260 1. McPhee JS, French DP, Jackson D, Nazroo J, Pendleton N, Degens H. Physical

261 activity in older age: perspectives for healthy ageing and frailty. Biogerontology

$262 \quad 2016 ; 17: 567-80$

263 2. Kodama S, Saito K, Tanaka S, et al. Cardiorespiratory fitness as a quantitative predictor of all-cause mortality and cardiovascular events in healthy men and women: a metaanalysis. Jama 2009;301:2024-35.

266

3. Stamatakis E, Lee IM, Bennie J, et al. Does Strength-Promoting Exercise Confer Unique Health Benefits? A Pooled Analysis of Data on 11 Population Cohorts With AllCause, Cancer, and Cardiovascular Mortality Endpoints. American journal of epidemiology 2018; 187:1102-12.

4. Bakker EA, Lee DC, Sui X, et al. Association of Resistance Exercise With the Incidence of Hypercholesterolemia in Men. Mayo Clinic proceedings 2018;93:419-28. 5. Giampaoli S, Ferrucci L, Cecchi F, et al. Hand-grip strength predicts incident

273 disability in non-disabled older men. Age and ageing 1999;28:283-8.

274 6. Rantanen T, Guralnik JM, Foley D, et al. Midlife hand grip strength as a predictor of old age disability. Jama 1999;281:558-60.

7. Onder G, Penninx BW, Ferrucci L, Fried LP, Guralnik JM, Pahor M. Measures of

277 physical performance and risk for progressive and catastrophic disability: results from the

278 Women's Health and Aging Study. The journals of gerontology Series A, Biological sciences and medical sciences 2005;60:74-9.

280 8. Rantanen T, Avlund K, Suominen H, Schroll M, Frandin K, Pertti E. Muscle strength as a predictor of onset of ADL dependence in people aged 75 years. Aging clinical and experimental research 2002; $14: 10-5$. 
A Meta-analysis of Prospective Cohort Studies. Journal of the American Medical Directors Association 2017; 18:551.e17-.e35.

10. Oksuzyan A, Demakakos P, Shkolnikova M, et al. Handgrip strength and its prognostic value for mortality in Moscow, Denmark, and England. PloS one 2017; 12:e0182684.

290

11. Celis-Morales CA, Welsh P, Lyall DM, et al. Associations of grip strength with cardiovascular, respiratory, and cancer outcomes and all cause mortality: prospective cohort study of half a million UK Biobank participants. BMJ (Clinical research ed) 2018;361:k1651. 12. Ridker PM, Rifai N, Cook NR, Bradwin G, Buring JE. Non-HDL cholesterol, apolipoproteins A-I and B100, standard lipid measures, lipid ratios, and CRP as risk factors for cardiovascular disease in women. Jama 2005;294:326-33.

13. Sin DD, Man SF. Why are patients with chronic obstructive pulmonary disease at increased risk of cardiovascular diseases? The potential role of systemic inflammation in chronic obstructive pulmonary disease. Circulation 2003;107:1514-9.

14. Tanaka T, Narazaki M, Kishimoto T. IL-6 in inflammation, immunity, and disease.

Cold Spring Harbor perspectives in biology 2014;6:a016295.

15. Du Clos TW. Function of C-reactive protein. Annals of medicine 2000;32:274-8.

16. Lowe GD. Fibrinogen assays for cardiovascular risk assessment. Clinical chemistry 2010;56:693-5.

17. Ernst E, Resch KL. Fibrinogen as a cardiovascular risk factor: a meta-analysis and review of the literature. Annals of internal medicine 1993;118:956-63. with established cardiovascular disease risk factors, inflammatory markers, and other 
characteristics: individual participant meta-analysis of 154,211 adults in 31 prospective studies: the fibrinogen studies collaboration. American journal of epidemiology 2007; 166:867-79.

19. Howren MB, Lamkin DM, Suls J. Associations of depression with C-reactive protein, IL-1, and IL-6: a meta-analysis. Psychosomatic medicine 2009;71:171-86.

20. Londhe P, Guttridge DC. Inflammation induced loss of skeletal muscle. Bone 2015;80:131-42.

21. Pedersen BK. Exercise-induced myokines and their role in chronic diseases. Brain, behavior, and immunity 2011;25:811-6.

22. Hamer M, Molloy GJ. Association of C-reactive protein and muscle strength in the English Longitudinal Study of Ageing. Age (Dordrecht, Netherlands) 2009;31:171-7. 23. Taaffe DR, Harris TB, Ferrucci L, Rowe J, Seeman TE. Cross-sectional and prospective relationships of interleukin-6 and C-reactive protein with physical performance in elderly persons: MacArthur studies of successful aging. The journals of gerontology Series A, Biological sciences and medical sciences 2000;55:M709-15.

24. Schaap LA, Pluijm SM, Deeg DJ, Visser M. Inflammatory markers and loss of muscle mass (sarcopenia) and strength. The American journal of medicine 2006;119:526.e917.

25. Steptoe A, Breeze E, Banks J, Nazroo J. Cohort profile: the English longitudinal study of ageing. International journal of epidemiology 2013;42:1640-8.

26. Craig R, Deverill C, Pickering K. Quality control of blood, saliva and urine analytes. Health survey for England 2004, Methodology and documentation Vol 2. London: The Information Centre; 2006:34-41. 
27. Irwin M, Artin KH, Oxman MN. Screening for depression in the older adult: criterion validity of the 10-item Center for Epidemiological Studies Depression Scale (CES-D). Archives of internal medicine 1999;159:1701-4.

28. Banks JA, Karlsen S, Oldfield Z. Socio-economic position. In: Marmot M, Banks JA, Blundell R, Lessof C, Nazroo J,. In: Marmot M, Banks JA, Blundell R, Lessof C, Nazroo J, eds. Health, wealth and lifestyles of the older population in England: The 2002 English Longitudinal Study of Ageing. London: Institute of Fiscal Studies; 2003.

29. Syddall HE, Westbury LD, Shaw SC, Dennison EM, Cooper C, Gale CR. Correlates of Level and Loss of Grip Strength in Later Life: Findings from the English Longitudinal Study of Ageing and the Hertfordshire Cohort Study. Calcified tissue international 2018;102:53-63.

30. Quan S, Jeong JY, Kim DH. The Relationship between Smoking, Socioeconomic Study. Epidemiology and health 2013;35:e2013001.

31. Smith L, White S, Stubbs B, et al. Depressive symptoms, handgrip strength, and weight status in US older adults. Journal of affective disorders 2018;238:305-10. cardiovascular events: potential mediating mechanisms. Circulation 2007;116:2110-8.

33. Sterne JA, Davey Smith G. Sifting the evidence-what's wrong with significance tests? BMJ (Clinical research ed) 2001;322:226-31.

34. Granic A, Davies K, Martin-Ruiz C, et al. Grip strength and inflammatory biomarker profiles in very old adults. Age and ageing 2017;46:976-82. between C-reactive protein and physical performance in older populations: results from the International Mobility in Aging Study (IMIAS). Age and ageing 2016;45:274-80. 
36. Kasapis C, Thompson PD. The effects of physical activity on serum C-reactive protein and inflammatory markers: a systematic review. Journal of the American College of Cardiology 2005;45:1563-9.

37. Visser M, Pluijm SM, Stel VS, Bosscher RJ, Deeg DJ. Physical activity as a determinant of change in mobility performance: the Longitudinal Aging Study Amsterdam. Journal of the American Geriatrics Society 2002;50:1774-81.

38. Schrager MA, Metter EJ, Simonsick E, et al. Sarcopenic obesity and inflammation in the InCHIANTI study. Journal of applied physiology (Bethesda, Md : 1985) 2007;102:91925.

39. Pedersen BK, Febbraio MA. Muscles, exercise and obesity: skeletal muscle as a secretory organ. Nature reviews Endocrinology 2012;8:457-65.

40. Visser M, Pahor M, Taaffe DR, et al. Relationship of interleukin-6 and tumor necrosis factor-alpha with muscle mass and muscle strength in elderly men and women: the Health ABC Study. The journals of gerontology Series A, Biological sciences and medical sciences 2002;57:M326-32.

41. Garcia-Hermoso A, Cavero-Redondo I, Ramirez-Velez R, et al. Muscular Strength as a Predictor of All-Cause Mortality in an Apparently Healthy Population: A Systematic Review and Meta-Analysis of Data From Approximately 2 Million Men and Women. Archives of physical medicine and rehabilitation 2018.

42. Perrig-Chiello P, Hutchison S. Health and well-being in old age: the pertinence of a gender mainstreaming approach in research. Gerontology 2010;56:208-13.

43. Cruz-Jentoft AJ, Baeyens JP, Bauer JM, et al. Sarcopenia: European consensus on definition and diagnosis: Report of the European Working Group on Sarcopenia in Older People. Age and ageing 2010;39:412-23. 
44. Studenski SA, Peters KW, Alley DE, et al. The FNIH sarcopenia project: rationale, study description, conference recommendations, and final estimates. The journals of gerontology Series A, Biological sciences and medical sciences 2014;69:547-58.

45. Berghella AM, Contasta I, Marulli G, et al. Ageing gender-specific "Biomarkers of Homeostasis", to protect ourselves against the diseases of the old age. Immunity \& ageing : I \& A $2014 ; 11: 3$.

46. Chupel MU, Direito F, Furtado GE, et al. Strength Training Decreases Inflammation and Increases Cognition and Physical Fitness in Older Women with Cognitive Impairment. Frontiers in physiology 2017;8:377.

47. Goodpaster BH, Park SW, Harris TB, et al. The loss of skeletal muscle strength, mass, and quality in older adults: the health, aging and body composition study. The journals of gerontology Series A, Biological sciences and medical sciences 2006;61:1059-64.

48. Roberts HC, Denison HJ, Martin HJ, et al. A review of the measurement of grip strength in clinical and epidemiological studies: towards a standardised approach. Age and ageing 2011;40:423-9.

49. Lawman HG, Troiano RP, Perna FM, Wang CY, Fryar CD, Ogden CL. Associations of Relative Handgrip Strength and Cardiovascular Disease Biomarkers in U.S. Adults, 20112012. American journal of preventive medicine 2016;50:677-83.

50. Whitney DG, Peterson MD. The Association Between Differing Grip Strength Measures and Mortality and Cerebrovascular Event in Older Adults: National Health and Aging Trends Study. Frontiers in physiology 2018;9:1871.

51. Tikkanen E, Gustafsson S, Amar D, et al. Biological Insights Into Muscular Strength: Genetic Findings in the UK Biobank. Scientific reports 2018;8:6451.

52. de Vreede PL, Samson MM, van Meeteren NL, Duursma SA, Verhaar HJ. Functional-task exercise versus resistance strength exercise to improve daily function in older 
405 women: a randomized, controlled trial. Journal of the American Geriatrics Society 2005;53:240610.

407

408 
Table 1. Baseline descriptive characteristics by sex specific handgrip tertiles.

\begin{tabular}{|l|l|l|l|}
\hline \multirow{2}{*}{ Baseline variable } & \multicolumn{3}{|c|}{ Sex specific handgrip tertilef } \\
\cline { 2 - 4 } & Low & Middle & High \\
\hline Age (yrs; mean, SD) & $70.6 \pm 10.3$ & $65.8 \pm 9.0$ & $62.3 \pm 7.8$ \\
\hline Smoking (\%) & 14.2 & 14.4 & 18.6 \\
\hline Vigorous physical activity (\%) & 18.8 & 29.3 & 41.2 \\
\hline Highest wealth quintile (\%) & 17.4 & 24.2 & 28.5 \\
\hline Longstanding illness (\%) & 66.0 & 49.9 & 41.7 \\
\hline Depressive symptoms (\%) & 18.8 & 11.9 & 10.2 \\
\hline Central obesity (\%) & 68.0 & 56.9 & 23.7 \\
\hline C-reactive protein (mg/L) $\dagger$ & $2.8(4.3)$ & $2.0(3.0)$ & $1.3(2.2)$ \\
\hline Fibrinogen (g/L)† & $3.3(0.9)$ & $3.2(0.9)$ & $3.0(0.8)$ \\
\hline
\end{tabular}

410 ‡ grip strength standardised for body weight values; lowest tertile ( $<0.42$ men, $<0.28$ women),

411 middle ( $0.42-0.52$ men, $0.28-0.37$ women), highest ( $>0.52$ men, $>0.37$ women).

$412+$ data are median (interquartile range) 
Table 2. Longitudinal association between grip strength and inflammatory markers over 8 years

follow up: English Longitudinal Study Ageing

\begin{tabular}{|c|c|c|}
\hline & \multicolumn{2}{|c|}{ Log C-reactive protein } \\
\hline & Model 1 & Model 2 \\
\hline Whole sample $(n=2705)$ & $-0.060(-0.092,-0.028)$ & $-0.049(-0.083,-0.015)$ \\
\hline $\operatorname{Men}(n=1211)$ & $-0.046(-0.087,-0.006)$ & $-0.038(-0.082,0.006)$ \\
\hline \multirow[t]{3}{*}{ Women $(n=1494)$} & $-0.068(-0.11,-0.030)$ & $-0.055(-0.095,-0.015)$ \\
\hline & \multicolumn{2}{|c|}{ Fibrinogen } \\
\hline & Model 1 & Model 2 \\
\hline Whole sample $(n=2624)$ & $-0.053(-0.077,-0.029)$ & $-0.044(-0.070,-0.019)$ \\
\hline Men $(n=1168)$ & $-0.041(-0.073,-0.009)$ & $-0.026(-0.060,0.008)$ \\
\hline Women ( $n=1456)$ & $-0.053(-0.081,-0.025)$ & $-0.046(-0.075,-0.016)$ \\
\hline
\end{tabular}

B $(95 \% \mathrm{Cl})$ coefficients per SD increase in grip strength standardised for body weight

Model 1 adjusted for age, sex and CRP or fibrinogen at baseline (sex adjustment excluded for sex specific models).

Model 2 adjusted for age, sex, CRP or fibrinogen at baseline, waist-hip-ratio, smoking, physical activity, depressive symptoms, chronic illness, wealth. 
Table 3. The extent to which baseline clinical and biological factors explain associations between handgrip and mortality

\begin{tabular}{|c|c|c|c|}
\hline & $\begin{array}{l}\text { Model } 1 \\
\text { HR }(95 \% \mathrm{Cl})\end{array}$ & $\begin{array}{l}\text { Model } 2 \\
\text { HR }(95 \% \mathrm{Cl})\end{array}$ & $\begin{array}{l}\text { Model } 3 \\
\text { HR (95\% Cl) }\end{array}$ \\
\hline $\begin{array}{l}\text { Whole sample } \\
\text { ( } n=5240 ; 650 \text { events) }\end{array}$ & $0.87(0.78,0.97)$ & $0.90(0.80,1.00)$ & $0.93(0.82,1.04)$ \\
\hline $\begin{array}{l}\text { Men } \\
\text { ( } n=2422 ; 355 \text { events) }\end{array}$ & $0.94(0.83,1.06)$ & $0.93(0.82,1.06)$ & $0.95(0.84,1.08)$ \\
\hline $\begin{array}{l}\text { Women } \\
\text { ( } n=2818 ; 295 \text { events) }\end{array}$ & $0.85(0.74,0.98)$ & $0.92(0.79,1.07)$ & $0.95(0.82,1.11)$ \\
\hline
\end{tabular}

Hazard ratio, $\mathrm{HR},(95 \% \mathrm{Cl})$ for risk of mortality per SD increase in grip strength standardised for body weight.

Model 1: adjusted for age, sex, wealth (sex adjustment excluded for sex specific models);

Model 2: further adjusted for clinical and behavioural covariates; smoking, physical activity, chronic illness, central obesity, depressive symptoms 